\title{
Optimization of the parameters of a partially regular microrelief by vibration rolling method
}

\author{
Valeriy Talamanov, Galina Kozenkova, Vladimir Kozenkov, Sergey Kondratyev, Evgeniy Heckert \\ Admiral Ushakov State Maritime University, 93 Lenin Ave., Novorossiysk, 353924, Russian Federation
}

\begin{abstract}
Experience of operating machines shows that their quality depends on the nature of the contact of mating parts. The irregular nature of the surface microrelief, which is due to traditional treatment methods used, arises difficulties in solving three main problems of microgeometry optimization: reliable, theoretically substantiated normalization, technological support, accurate measurement and control. This determined the direction in solving the problem of increasing the accuracy and reliability of the surface quality normalization, that is the microrelief normalization. At present, there are a large number of technological methods for surface treatment aimed to form a regular microrelief on it. One of the most common and studied methods for the regular microrelief formation is the vibration rolling method based on thin plastic deformation of the surface metal layers and a complex relative displacement of the treated surface and the deforming element. Significant progress in the surface quality normalization was achieved after the introduction of GOST 24773-81, the standard for regular microreliefs. For example, the nomenclature of parameters and characteristics of partially regular microreliefs includes the relative area occupied by regular inhomogeneities $F_{H} . F_{H}$ is a value expressed as a percentage of the area occupied by regular inhomogeneities to the area of the treated surface. If $F_{H}$ is determined for the $2 A \cdot S_{k}$ area within the boundaries of a microrelief element at different axial steps of regular inhomogeneities, the microgeometry of a partially regular microrelief can be described ambiguously. To avoid this, it is necessary to consider the multiplicity of the ratio of the amplitude $A$ to the axial step $S_{o}$.
\end{abstract}

\section{Introduction}

Experience of operating machines shows that their quality depends on the nature of the contact of mating parts [4]. Therefore, the focus is on optimization of the contact surface quality. Among different characteristics such as roughness and waviness, physical, mechanical, chemical properties and microstructure of the surface layer, which determine the surface quality of machine parts, optimization of the microgeometry of the contact surfaces is one of the most important. Requirements for the parameters of microgeometry are based on their relationship with the functional indicators of machine parts. The values of these parameters can be calculated using theoretical or empirical equations for the relationship between the operational characteristics of machine parts and their junctions and the quality characteristics of surfaces. The complexity of solving the problem of optimizing the microgeometry of contact surfaces is aggravated by the irregular nature of the surface microrelief, which is due to traditional treatment methods used.

This microrelief causes difficulty in solving three main problems of microgeometry optimization: reliable, scientifically grounded normalization, technological support, accurate measurement and control.
This determined the direction in solving the problem of increasing the accuracy and reliability of the surface quality normalization, that is the microrelief normalization.

A complex technological problem is solved in various ways, both in relation to the method of influencing the processed material, and in relation to the principle of regularization of the microrelief.

The complexity of the task is compounded not only by the fact that it is necessary to create a regular microrelief on the surfaces of various materials, but also to "manage" it very subtly and within large limits, i.e. to vary the values of all its parameters: height, step and area. At the same time, the variation of the value of each parameter should be independent, i.e. such that when the value of one parameter changes, the values of the others remain unchanged.

For example, when changing the height of irregularities, their pitch should not change, as it happens when turning and grinding.

Almost all known methods of forming regular microreliefs do not meet these requirements.

At present, there are a large number of technological methods for surface treatment aimed to form a regular microrelief on it [2]. One of the most common and studied methods for the regular microrelief formation is the vibrational rolling method developed by Professor of LITMO Yu. G. Shneider, which is based on thin plastic

$\overline{\text { *Corresponding author: talamanov@ mail.ru }}$ 
deformation of the surface metal layers and a complex relative displacement of the treated surface and the deforming element. [6].

Recently, a large number of studies, laboratory and operational tests of various machine parts and devices with a regular microrelief have been carried out in the Russian Federation and abroad, which revealed their new operational characteristics higher than those of parts treated by traditional methods. [3].

\section{Problem statement}

Normalization of the microgeometry should provide a complete description to ensure its optimization.

The microrelief inhomogeneity complicates the control of the geometric parameters of the surface quality and their standardization. Thus, when standardizing in accordance with GOST 2789-73, it is necessary to take average values as the microrelief parameters, for example, $R_{a}$ - arithmetic mean of the profile deviation; $R_{z}-$ height of the profile inhomogeneities at ten points; $S_{m}$ - average value of the step of the profile inhomogeneities within the base length; $S$ - average value of the steps of the profile projections that are within the base length. This complicates the measurement and control of the microgeometric characteristics of the real surface. For a more complete description of the surface roughness, the harmonic analysis is used, when the surface profilogram can be represented as a finite set of harmonics [4]. Significant progress in the surface quality normalization was achieved after the introduction of GOST 24773-81, the standard for regular microreliefs.

A high degree of homogeneity of the microgeometry over the entire surface formed by vibration rolling characterizes its microrelief using geometric parameters that remain unambiguous over the entire working surface. For example, the nomenclature of parameters and characteristics of partially regular microreliefs includes the relative area occupied by regular inhomogeneities $\mathrm{F}_{\mathrm{H}} . \mathrm{F}_{\mathrm{H}}$ is a value expressed as a percentage of the area occupied by regular inhomogeneities to the area of the treated surface. The $\mathrm{F}_{\mathrm{H}}$ parameter determined for the $2 A \cdot S_{k}$ area is of particular interest.

\section{Materials and methods}

The vibration rolling method is used to improve the operational characteristics of machine parts.

The vibration rolling method is based on two principles: refusal of cutting, the use of thin plastic deformation, and complication of the kinematic processing [6]. The kinematic dependencies that characterize the vibration rolling method are much more complicated than those for ball rolling.

Figure 1 presents the parameters that determine the mode of vibration rolling of cylindrical surfaces: nw workpiece rotation frequency, rpm; $S$ - feed of the deforming element per a turn of the workpiece, $\mathrm{mm} / \mathrm{rev}$; nd.el. - number of oscillations of the deforming element, min; A - oscillation amplitude, $\mathrm{mm}$; $\mathrm{dw}$ - the workpiece diameter, $\mathrm{mm}$; $\mathrm{P}$ - force of the deforming element indentation, N. The deforming element can be a ball, spherical diamond, carbide or other tip.

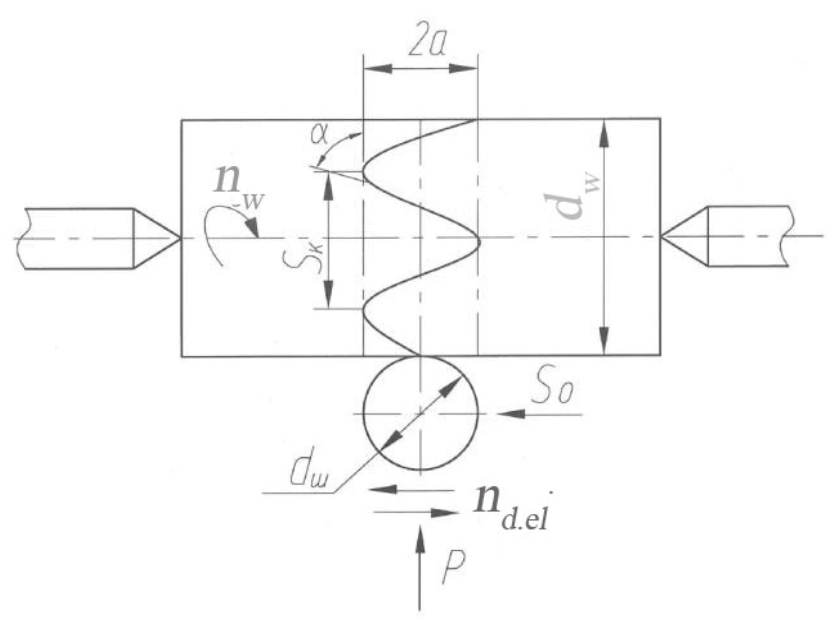

Fig 1. Parameters of the vibration rolling mode.

Figure 2 presents a schematic diagram of the line vibration rolling of the flat surface. The parameters of the vibration rolling mode when treating flat surfaces include: longitudinal $\left(S_{l}\right)$ and transverse $\left(S_{t}\right)$ feed of the workpiece, the oscillation frequency of the deforming element $\left(n_{\text {d.el }}\right)$ and the oscillation amplitude of the deforming element $(A)$.

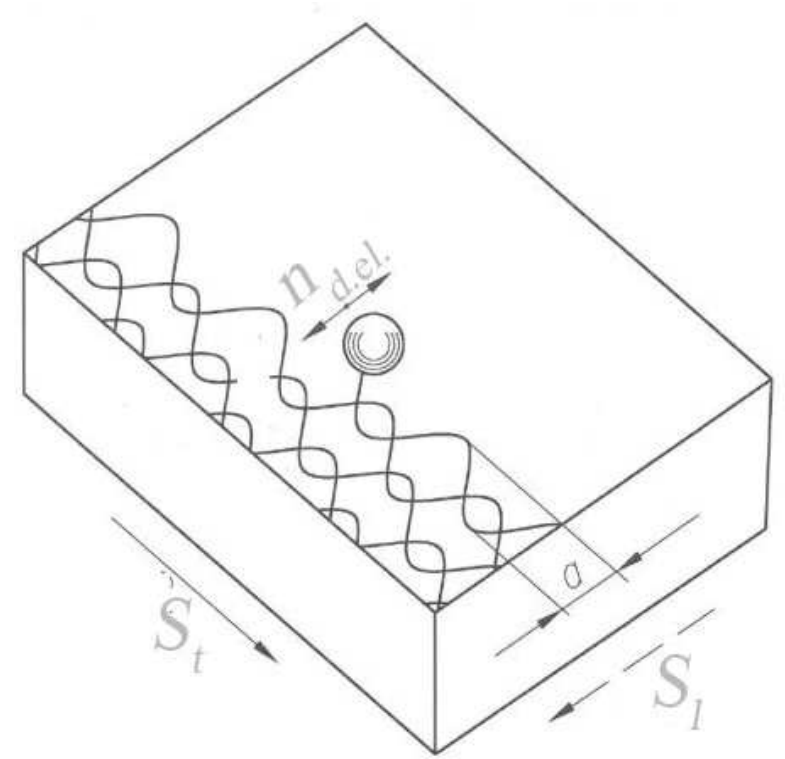

Fig. 2 Schematic diagram of line vibration rolling of the flat surface

The control of the formation of a regular or partially regular microrelief implies varying the ratio of motion speeds of the workpiece and the deforming element.

In terms of the nature and density of the arrangement of the sinusoidal grooves, the resulting microrelief is divided into four types: grooves do not contact; contact; intersect; superimpose to form a microrelief without the original surface areas. Figure 3 shows types of partially regular microreliefs with continuously or discretely 
located indentation with remained areas of the original surface.

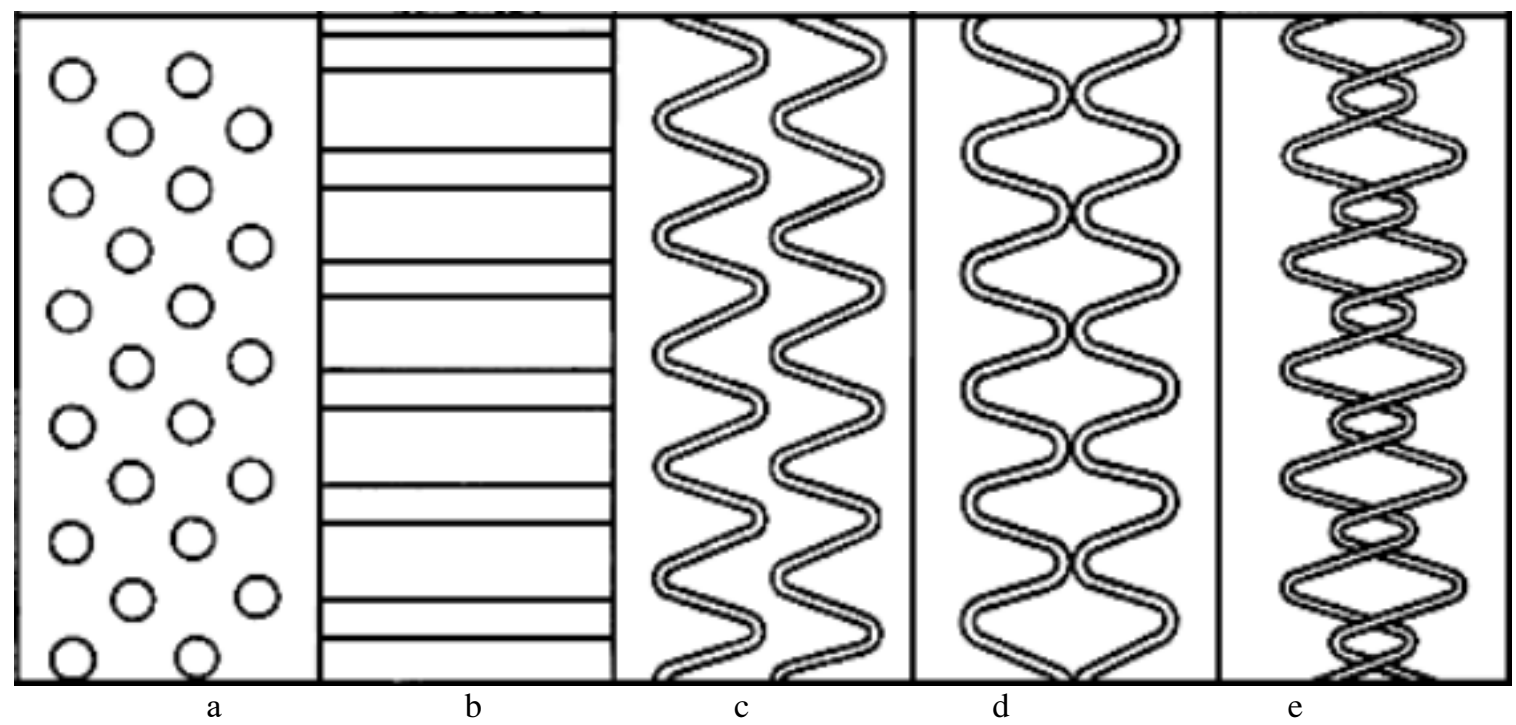

Fig. 3. Types of partially regular microreliefs:

$\mathrm{a}$ - staggered arrangement of regular inhomogeneities; $\mathrm{b}$ - annular arrangement of regular inhomogeneities; $\mathrm{c}-$ no intersection of regular inhomogeneities;

$\mathrm{d}$ - incomplete intersection of regular inhomogeneities; $\mathrm{d}$ - complete intersection of regular microreliefs

In accordance with GOST 24773-81, the nomenclature of parameters and characteristics of partially regular microreliefs includes:

$\mathrm{h}$ - depth (height) of regular inhomogeneities;

$\mathrm{F}_{\mathrm{k}}$ - relative area occupied by regular inhomogeneities;

$\mathrm{S}_{\mathrm{o}}$ - circular step of regular inhomogeneities;

A - amplitude of a continuous regular inhomogeneity.

For partially regular microreliefs, the following limits are established for the numerical values of their parameters: $h=0.025 / 1600 \mu \mathrm{m}, F_{k}=10 / 90 \%, S_{o}$ and $S_{k}=0.05 / 200 ; A=0.5 / 5$.

Partially regular microreliefs formed by the vibration rolling method differ from traditional ones formed by all other treatment methods by the homogeneity of all geometric parameters, which are functionally related to the mode parameters, and provide the opportunity to normalize the microrelief parameters by setting them on the basis of analytical calculations through the parameters of the vibration rolling mode.

It should be noted that the values of the regular microrelief parameters are reproduced with high accuracy at the appropriate vibration rolling mode and the need to measure them using instruments is eliminated. The control of the parameters of the vibration rolling mode is sufficient.

At present, the vibration rolling method is being developed through the increased scope of application, and the following technological problems are being solved:

- stabilization of the process in terms of the depth and shape of the resulting sinusoidal groove;

- creation of methods for calculating the vibration rolling parameters and the microrelief characteristics depending on the task set;
- creation of effective technological equipment for vibration rolling using $\mathrm{CNC}$ machines and flexible production systems [1].

\section{Optimization of the parameters of a partially regular microrelief}

The $\mathrm{F}_{\mathrm{H}}$ parameter of a partially regular microrelief most fully determines almost all the operational characteristics of surfaces and, first of all, the actual contact area of the solid surface with another surface, the oil absorption of the surface, the ability to prevent wear products in the sinusoidal groove volume from carrying of the contacting friction pairs to the surface.

According to GOST 24773-81, $\mathrm{F}_{\mathrm{H}}$ is a value expressed as a percentage of the area occupied by regular inhomogeneities to the area of the treated surface. It is of interest to determine $\mathrm{F}_{\mathrm{H}}$ for the $2 A \cdot S_{k}$ area within the boundaries of the microrelief element at various axial steps $S$ of regular inhomogeneities.

Consider the elements of a partially regular microrelief formed by vibration rolling presented in Fig. 4 [5]. 


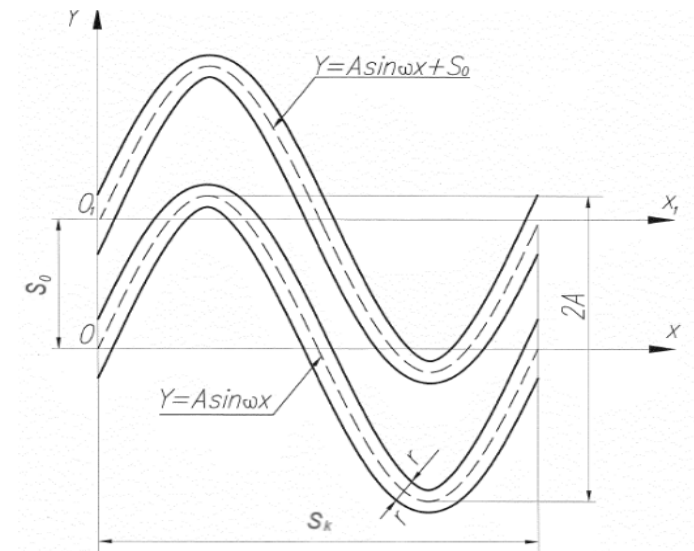

Fig. 4. Elements of a partially regular microrelief $\mathrm{S}_{\mathrm{o}}$ - axial step of regular inhomogeneities;

$\mathrm{S}_{\mathrm{k}}$ - circular step of regular inhomogeneities;

$\mathrm{A}-$ amplitude of a continuous regular inhomogeneity.

The trajectory of the deforming element center is described by the equation

$$
y=A \cdot \operatorname{Sin} \omega x
$$

To simplify the calculation, assume that the upper and lower boundaries of the sinusoidal groove are described by the equation

$$
y=A \sin \omega x \pm r
$$

where $r$ - radius of the imprint of the deforming element on the plane.

For the adjacent sinusoidal groove displaced by the value of the axial step of regular inhomogeneities $S_{o}$, the trajectory of the deforming element center has the form

$$
y=A \sin \omega x \div S_{o}
$$

Set the XOY coordinate system, then the equations for the trajectories of the deforming element center have the form

$$
y_{j}=A \sin \omega x+j S_{o}, \quad \mathrm{j}=0 ; \pm 1 ; \quad \pm 2
$$

Consider the parameter $F_{H}$ on the $S_{k} \cdot 2 A$ area, where $S_{k}=2 \pi / \omega$ at different axial steps of regular inhomogeneities $\mathrm{S}_{\mathrm{k}}$. Let us have an area

$$
\begin{array}{r}
\Pi_{0}=\left[(0,-\mathrm{A}),(0, \mathrm{~A}),\left(\mathrm{S}_{\mathrm{k}}, \mathrm{A}\right),\left(\mathrm{S}_{\mathrm{k}}-\mathrm{A}\right)\right] \\
\text { at } \mathrm{S}=2 \mathrm{~A} / \mathrm{\kappa}, \mathrm{\kappa}=1,2, \ldots,[\mathrm{A}-2 \mathrm{r}]
\end{array}
$$

The exact number $\kappa$ of the sinusoidal grooves is located on the area $\Pi_{0}$ and due to symmetry on each area

$$
\left.\Pi_{\tau}=[0,-\mathrm{A}+\tau),(0, \mathrm{~A}+\tau),\left(\mathrm{S}_{\mathrm{k}}, \mathrm{A}+\tau\right),\left(\mathrm{S}_{\mathrm{k}},-\mathrm{A}+\tau\right)\right]
$$

Hence, as a result, the parameter $F_{H}$ is constant on any of the areas $\Pi_{\tau}$.

Previously, calculations were carried out to confirm the ambiguity of the parameter $F_{H}$ at different axial step $S_{o}$ on the $2 A \cdot S_{k}$ area at $2 A /(k+1)<S_{o}<2 A / k$ when the symmetry is violated [5].
Analysis of the numerical values of the axial step $S_{o}$ and the amplitude of regular inhomogeneity $A$ specified in the standard [7] shows that not all numerical values can provide the condition $S_{o}=2 \mathrm{~A} / \mathrm{k}$

This necessitates further improvement of GOST 24773-81.

\section{Conclusion and recommendation}

5.1 The parameter $F_{H}$ of a partially regular microrelief most fully determines practically all the operational characteristics of surfaces and, first of all, the actual contact area of a solid surface with another surface, oil absorption of the surface, the ability to prevent foreign particles from carrying to the contact surface.

5.2 An important parameter $F_{H}$, the relative area occupied by regular inhomogeneities, ambiguously describes the microgeometry of a regular microrelief with the ratio of the amplitude parameter $A$ and the axial step $S$.

$$
2 A /(\kappa+1)<S<2 A / \kappa
$$

5.3 To provide an unambiguous description of the microgeometry of a regular microrelief using the $F_{H}$ parameter, it is necessary to consider the multiplicity of the ratio of the amplitude $A$ and the axial step $S$.

$$
S=2 A / \kappa, \kappa=1,2, \ldots,[A-2 r]
$$

5.4 Analysis of the numerical values of the axial step $S_{o}$ and the amplitude of a regular inhomogeneity $A$ specified in the standard shows that not all numerical values can provide the condition $S_{o}=2 \mathrm{~A} / \mathrm{k}$.

This necessitates further improvement of GOST 24773-81.

\section{References}

1. Yu. P. Kuzmin Journal of Instrument Engineering 53(8), (2010)

2. S. A. Kurguzov, D. N. Krasnoperov, I. A. Krasnoperova, I. V. Yakunina, E. A. Burmistrova Scientific Papers SWorld 6(4), (2014)

3. A. A. Kabatov Open information and computer integrated technologies 58, 49-54, (2013)

4. A. S. Pronikov Parametric reliability of machines (M.: Publishing House of the Bauman Moscow State Technical University, 2002)

5. I. S. Katryuk, B. A. Stefan, V. N. Talamanov, G. L. Kozenkova Proceedings of the 11th National Scientific and Technical Conference "Mechanisms for ensuring the competitiveness of the transport complex of the South of Russia" (Novorossiysk, RIO State Medical University named after Admiral F. F. Ushakov, 2018)

6. Yu. G. Schneider Operational characteristics of parts with regular microreliefs. (L.: Mashinostroenie, 1982).

7. GOST 24773-81 Surfaces with regular 
microrelief. Classification, parameters and characteristics. 